\title{
Méthode énergétique inverse appliquée à la détection de sources acoustiques
}

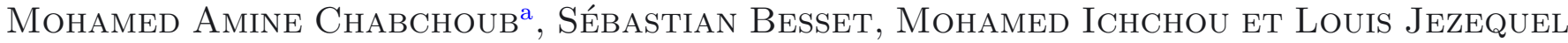 \\ Laboratoire de Tribologie et Dynamique des Systèmes, CNRS-UMR 5513, Équipe de Dynamique des Structures et des Systèmes \\ (D2S), École Centrale de Lyon, Université de Lyon, 36 avenue Guy de Collongue, 69134 Écully, France
}

Reçu le 30 septembre 2008, accepté le 18 décembre 2008

\begin{abstract}
Résumé - La qualité sonore des véhicules constitue aujourd'hui un critère important de conception. Dans ce cadre, la détection de sources acoustiques est une problématique majeure. En effet, si l'analyse du bruit dans une cavité peut être traitée de manière classique (FEM, BEM...), les problèmes inverses posent souvent des problèmes liés à la taille des calculs effectués ou au conditionnement des matrices à inverser. Nous nous proposons ici d'étudier une méthode énergétique simplifiée (MES) qui présente la particularité d'être avantageusement inversible. Le but de la méthode proposée est de parvenir à localiser des sources acoustiques surfaciques à partir de mesures effectuées dans une cavité.
\end{abstract}

Mots clés : Source acoustique / bruit / problème inverse / méthode énergétique simplifiée (MES) / hautes fréquences

\begin{abstract}
Inverse energy method applied to the acoustic sources detection. The sound quality of the vehicles constitutes today a significant criterion of design. Within this framework, the detection of acoustic sources is a major problem. In fact, if the noise analysis in a cavity can be treated in a traditional way (FEM, BEM...), inverse methods often generate problems related to calculation size carried out or the conditioning of the matrices to be inversed. We propose to study a simplified energy method (SEM) which has the effect of being advantageously inversed. The suggested method objective is to locate surface acoustic sources starting from measurements taken in a cavity.
\end{abstract}

Key words: Acoustic source / noise / inverse problem / simplified energy method (SEM) / high frequency

\section{Introduction}

Dans le domaine des basses fréquences, les méthodes d'éléments-finis et d'éléments-finis de frontière permettent de prédire correctement les niveaux vibratoires et acoustiques induits par différents types d'excitations. Malheureusement, lorsque le domaine fréquentiel d'analyse augmente, la mise en œuvre de la méthode des éléments-finis se trouve confrontée à l'augmentation excessive des degrés de liberté entraînant un coût de calcul prohibitif.

Ainsi, l'enjeu principal présenté dans cet article est d'utiliser une méthode de calcul appelée méthode énergétique simplifiée pour prédire le comportement

\footnotetext{
a Auteur pour correspondance :

mohamed.chabchoub@ec-lyon.fr
}

vibroacoustique dans le domaine des moyennes et hautes fréquences.

Plusieurs recherches sont faites pour élaborer et développer cette méthode afin de permettre de localiser les sources acoustiques surfaciques en utilisant la MES Inverse. Parmi ces recherches, on peut citer $[1,2]$ qui décrivent l'évolution de l'utilisation de l'équation de transport de l'énergie pour fonder la MES en présentant son cadre général d'application et de ses différentes approches.

La méthode énergétique simplifiée inverse permet alors de localiser les sources acoustiques surfaciques dans une cavité et de bien quantifier la valeur de la puissance injectée de chaque source. Elle permet donc d'isoler les sources acoustiques nuisibles afin de réduire le bruit dans la cavité étudiée. 
Dans cet article, on rappelle tout d'abord le principe de la MES en présentant ses différentes hypothèses et approches qui dépendent de différentes variantes qui sont proposées suivant que les champs sont décomposés en ondes planes ou en rayons. On présente ensuite les résultats trouvés en utilisant la MES pour localiser les sources acoustiques en évoquant les sensibilités de ces résultats à l'intrusion d'erreurs de mesure, à la distance entre les microphones et à la distance entre surfaces et microphones. Enfin, on valide ces résultats par d'autres résultats obtenus en alimentant la MES inverse par des données FEM.

\section{Méthode énergétique simplifiée}

La méthode énergétique simplifiée est une méthode de calcul vibroacoustique dans le domaine des moyennes et hautes fréquences. Et comme l'indique son nom, elle est basée sur un modèle énergétique, propagatoire et local. Les variables utiles à cette description énergétique prennent la forme de la densité d'énergie $W$ et du vecteur densité de flux $I$ appelée flux d'énergie ou encore intensité en acoustique. Ces champs sont la plupart du temps exprimés à partir de moyennes quadratiques de variables cinématiques telles que la pression et la vitesse acoustique, d'où leur nom de variables quadratiques. Différentes variantes sont alors proposées, suivant que les champs sont décomposés en ondes planes ou en rayons, et suivant le modèle de réflexion utilisé. En effet, la MES différentielle $[3,4]$ considère un champ d'ondes planes, la MES fonctionnelle et la MES intégrale [5,6] considèrent un champ de rayons, alors que la MES différentielle mixte [2] considère un champ direct de rayons et un champ réverbéré d'ondes planes.

La MES fonctionnelle utilise un modèle de réflexion spéculaire et la MES intégrale un modèle de réflexion diffuse. La MES différentielle repose en fait sur une équation de diffusion, et la MES intégrale se révèle comme une méthode de radiosité. C'est cette dernière méthode qui nous intéresse plus particulièrement.

\subsection{Hypothèses de la MES}

L'équation de base des modèles MES est le bilan de puissance élémentaire d'une portion du système. En se basant sur [1] et pour écrire cette équation, nous nous plaçons sous l'hypothèse H1 qui se base sur un système linéaire, isotrope, en régime stationnaire et à la pulsation $\omega$. Le bilan de puissance local s'écrit alors sous la forme :

$$
\nabla I+\Pi_{\mathrm{diss}}=\Pi_{\mathrm{inj}}
$$

Les grandeurs désignées par $\Pi$ sont des densités de puissance. $\Pi_{\mathrm{inj}}$ représente la puissance injectée et $\Pi_{\mathrm{diss}}$ désigne la puissance dissipée sur laquelle nous faisons une nouvelle hypothèse $\mathrm{H} 2$ qui s'appuie sur un modèle d'amortissement hystérétique. La puissance dissipée $\Pi_{\text {diss }}$ sera ici proportionnelle à l'énergie totale $W$. Cette proportionnalité s'écrit en acoustique sous la forme :

$$
\Pi_{\mathrm{diss}}=m c W
$$

où $m$ est le coefficient d'absorption et $c$ est la vitesse du son.

L'équation de bilan de puissance (1) s'écrit alors :

$$
\nabla I+m c W=\Pi_{\mathrm{inj}}
$$

On considère exclusivement que les ondes sont propagatives et que cette condition constitue l'hypothèse H3. On néglige donc les ondes dites de champ proche, et notamment les ondes évanescentes qui restent cantonnées au niveau des discontinuités, et dont l'étendue spatiale diminue avec la fréquence. La relation fondamentale qui relie l'intensité et le flux d'énergie s'écrit alors :

$$
I=c W u
$$

La quatrième hypothèse $\mathrm{H} 4$ constitue sans doute l'hypothèse la plus importante dans laquelle les interférences entre les ondes propagatives sont négligées. En fait, l'hypothèse de décorrélation des ondes revient à négliger les effets de phase, qui sont responsables du comportement modal des systèmes. Les modèles énergétiques ne donneront par conséquent pas d'information modale, et cette perte d'information est associée à un gain numérique certain.

\subsection{MES intégrale}

La méthode intégrale applique les hypothèses de décorrélation aux champs propagatifs issus d'une source ponctuelle dans le milieu infini de la dimension du système considéré : il s'agit d'ondes planes en 1D, cylindriques en $2 \mathrm{D}$ et sphériques en 3D. En coordonnées radiales, et d'après [7-11], (1) s'écrit :

$$
\frac{1}{r^{n-1}} \frac{\mathrm{d}}{\mathrm{d} r}\left(r^{n-1} I\right)+m c W=\delta(0)
$$

où $r$ est la variable radiale de l'onde, $n$ est la dimension de l'espace et $\delta$ désigne la distribution de Dirac.

Les solutions de cette équation sont appelées noyaux de Green énergétiques et on note $G$ le noyau de densité d'énergie et $H$ le noyau d'intensité.

$$
G(r)=\frac{1}{\gamma_{n} c} \frac{\mathrm{e}^{-m r}}{r^{n-1}} \quad H(r)=\frac{1}{\gamma_{n}} \frac{\mathrm{e}^{-m r}}{r^{n-1}} u_{r}
$$

L'hypothèse de décorrélation est utilisée pour appliquer le principe de superposition linéaire aux quantités énergétiques. Le champ total est par la suite construit comme la superposition des composantes de champ direct provenant des sources volumiques à l'intérieur du système $\Omega$ et des composantes réverbérées correspondant aux sources de surface localisées sur la frontière $\partial \Omega$, ces différents types de sources sont présentés dans la figure 1. 


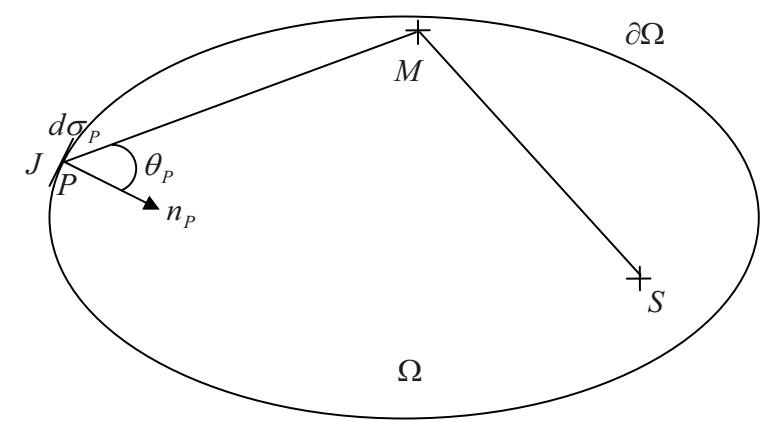

Fig. 1. Domaine $\Omega$ avec sources de surface de radiosité $J$.

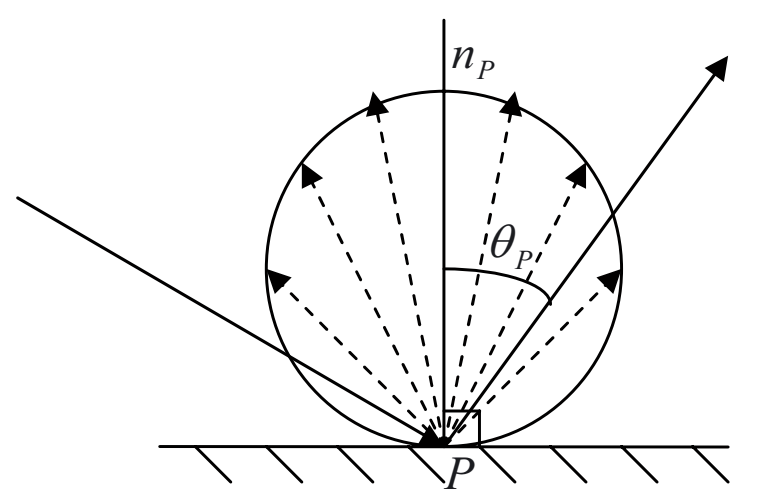

Fig. 2. Réflexion diffuse.

On désigne par $\varepsilon$ la puissance volumique des sources primaires et par $i_{+}$l'intensité spécifique attachée aux sources secondaires. Avec les notations de la figure 1, et à l'aide des noyaux de l'équation (6), la densité d'énergie et l'intensité en un point $\mathrm{M}$ sont respectivement données par :

$$
\begin{aligned}
W(M)= & \int_{\Omega} \varepsilon\left(S, u_{S M}\right) G(S, M) \mathrm{d} \tau_{S} \\
& +\int_{\partial \Omega} i_{+}\left(P, u_{P M}\right) G(P, M) \mathrm{d} \sigma_{P} \\
I(M)= & \int_{\Omega} \varepsilon\left(S, u_{S M}\right) H(S, M) \mathrm{d} \tau_{S} \\
& +\int_{\partial \Omega} i_{+}\left(P, u_{P M}\right) H(P, M) \mathrm{d} \sigma_{P}
\end{aligned}
$$

L'approche intégrale postule une réflexion diffuse, l'énergie est émise selon le cosinus de la direction d'émission avec la normale à la frontière $n_{P}$, repéré par l'angle $\theta_{P}$ (Fig. 2). Contrairement à la MES différentielle, nous conservons ici l'information de l'incidence du flux, la directivité dite de Lambert n'intervenant que sur l'énergie émise.

Conformément à la notion de radiosité, on écrit $i_{+}\left(P, u_{P M}\right)$ comme le produit de la radiosité $J(P)$ et d'un terme de directivité $D\left(u_{P M}\right)=D\left(\theta_{P}\right)$ proportionnel à $\cos \theta_{P}$. Cette directivité doit être normalisée de manière à ce que son intégration sur toutes les directions permette de retrouver l'angle solide. Ainsi :

En 2D : $\int_{0}^{\pi} \cos \theta \mathrm{d} \theta=2$ et $\gamma_{2}=2 \pi$ donc $D_{2 D}(\theta)=\pi \cos \theta$

En 3D :

$$
\begin{aligned}
\int_{\varphi=0}^{2 \pi} \int_{\theta=0}^{\frac{\pi}{2}} \cos \theta \sin \theta \mathrm{d} \theta \mathrm{d} \varphi= & \pi \text { et } \gamma_{3}=4 \pi \\
& \quad \text { donc } D_{3 D}(\theta)=4 \cos \theta
\end{aligned}
$$

On reprend alors les relations (7) et (8) pour écrire :

$$
\begin{aligned}
W(M)= & \int_{\Omega} \varepsilon\left(S, u_{S M}\right) G(S, M) \mathrm{d} \tau_{S} \\
& +\int_{\partial \Omega} 4 \cos \theta_{P} J(P) G(P, M) \mathrm{d} \sigma_{P} \\
I(M)= & \int_{\Omega} \varepsilon\left(S, u_{S M}\right) H(S, M) \mathrm{d} \tau_{S} \\
& +\int_{\partial \Omega} 4 \cos \theta_{P} J(P) H(P, M) \mathrm{d} \sigma_{P}
\end{aligned}
$$

Les sources de frontière constituent alors les inconnues du problème à résoudre. Elles décrivent le champ réverbéré et sont calculées en appliquant le bilan de puissance aux interfaces. Exprimant le flux incident de part et d'autre d'une interface, l'amplitude de la source secondaire correspond à la somme des puissances transmises et réfléchies, chacune décomposée en contributions directe et réverbérée. Le calcul MES est réalisé à partir d'une discrétisation en $N_{\mathrm{e}}$ facettes de la surface définissant la cavité. Dans une bande de fréquence donnée et pour un nombre de points de calcul $N$, on cherche le vecteur des flux entrants par l'équation :

$$
\left\{\varphi^{\text {entr }}\right\}=[S]^{-1}\{y\}
$$

où $\{y\}$ est le vecteur rassemblant les valeurs de champ comme suit :

$$
\{y\}=\left\{\begin{array}{l}
\{W\} \\
\{I\}
\end{array}\right\}
$$

et $[S]$ est la matrice de taille $\left(2 N, N_{\mathrm{e}}\right)$ rassemblant les opérateurs de sensibilité :

$$
[S]=\left[\left[\begin{array}{l}
S^{W} \\
{\left[S^{I}\right]}
\end{array}\right]\right]
$$

\section{Résultats MES}

Dans cette partie, on va tout d'abord valider la MES inverse en évoquant les sensibilités de ces résultats à l'intrusion d'erreurs de mesure, à la distance entre les microphones et à la distance entre surfaces et microphones. 

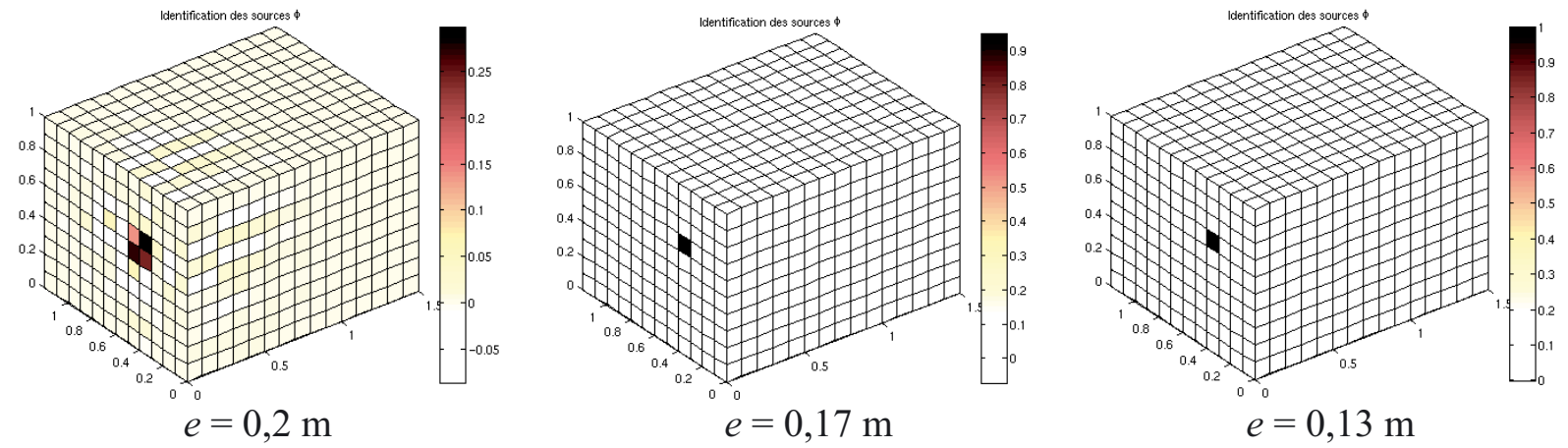

Fig. 3. Sensibilité à l'écartement entre les microphones.
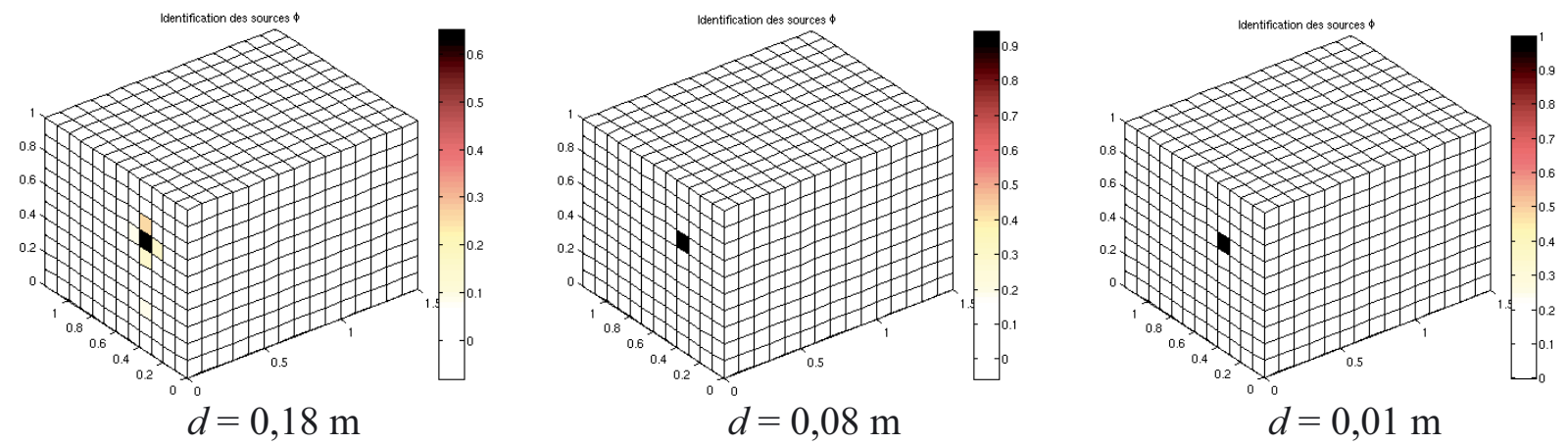

Fig. 4. Sensibilité à la distance entre les surfaces et les microphones.

Ensuite, on valide ces résultats par d'autres résultats obtenus en alimentant la MES inverse par des données FEM.

\subsection{Validation de la MES inverse}

Dans cette section, on étudie la faisabilité de la MES inverse, sa sensibilité à la disposition des données collectées par les microphones, puis sa robustesse après l'intrusion d'erreurs au niveau des données.

La validation MES inverse consiste à prendre des sources connues, établir le calcul du champ acoustique par la MES directe, puis prélever des mesures dans certains points microphones, ensuite appliquer la MES inverse et voir si l'on retrouve les sources de départ. On cherche dans ce qui suit à valider la MES inverse pour une source surfacique placée aux coordonnées $(0,0,39,0,66)$ appartenant à la surface d'une cavité de forme cubique de dimensions $1,5 \times 1,2 \times 1$ mètres cubes maillée par 900 mailles surfaciques. La puissance injectée au niveau de la source est de $1 \mathrm{~W} \cdot \mathrm{m}^{-2}$ et les surfaces possèdent une absorption de 0,3 . Les microphones sont uniformément répartis sur les six surfaces d'un cube se trouvant à l'intérieur de notre cavité. On pose alors $d$ la distance entre les surfaces de la cavité et les microphones et de l'écartement entre les microphones.

On va tester ainsi la sensibilité de la MES inverse à l'écartement entre les micros. Les résultats sont présentés dans la figure 3 .
Il apparaît donc que la source sera bien localisée et quantifiée (valeur exacte de la puissance injectée trouvée) pour des valeurs de $e$ inférieures à $0,13 \mathrm{~m}$.

On teste aussi la sensibilité à la distance entre les surfaces et les microphones dont les résultats sont présentés dans la figure 4.

S'éloignant d'une distance inférieure à $10 \mathrm{~mm}$ de la surface, ça nous permet de trouver la bonne valeur de puissance injectée ainsi que le bon endroit de la source.

Pour déterminer la robustesse de la MES inverse, on teste sa sensibilité aux intrusions d'erreurs de calcul. On ajoute donc un pourcentage de la valeur mesurée comme erreur à chaque mesure. Les résultats trouvés suite à l'introduction de perturbations de mesure de $10 \%$, $30 \%$ et $90 \%$ présentés par la figure 5 montrent la possibilité d'identifier et de quantifier la source même avec une intrusion d'erreur qui est égale à $90 \%$. On a choisi pour ces résultats un écartement de $3 \mathrm{~cm}$ et une distance entre surfaces et microphones de $1 \mathrm{~cm}$.

On va étudier une autre fois la sensibilité à l'écartement $e$ et à la distance $d$ après l'intrusion d'une erreur de $10 \%$. Ces résultats sont résumés dans les figures 6 et 7 .

On remarque alors une diminution de la valeur maximale de $e$ qui permette à la fois de bonnes identification et quantification de la source de $13 \mathrm{~cm}$ sans intrusion d'erreur à $10 \mathrm{~cm}$ à la suite de l'intrusion.

On remarque aussi une diminution de la valeur maximale de $d$ qui permet à la fois de bonnes identification 


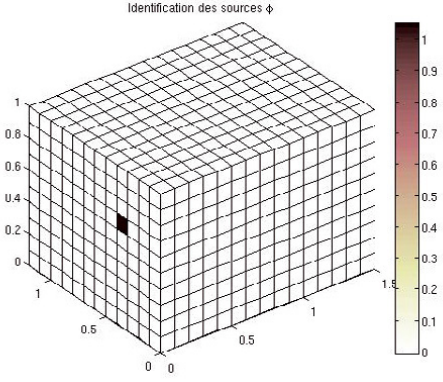

$10 \%$

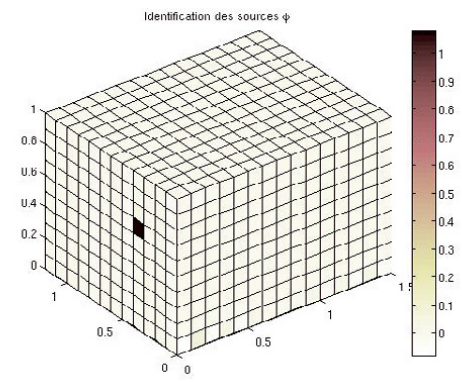

$30 \%$

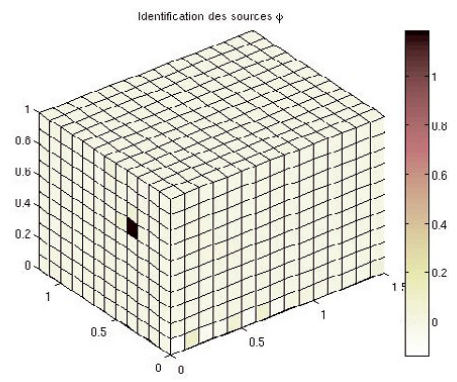

$90 \%$

Fig. 5. Résultats des perturbations de mesure.
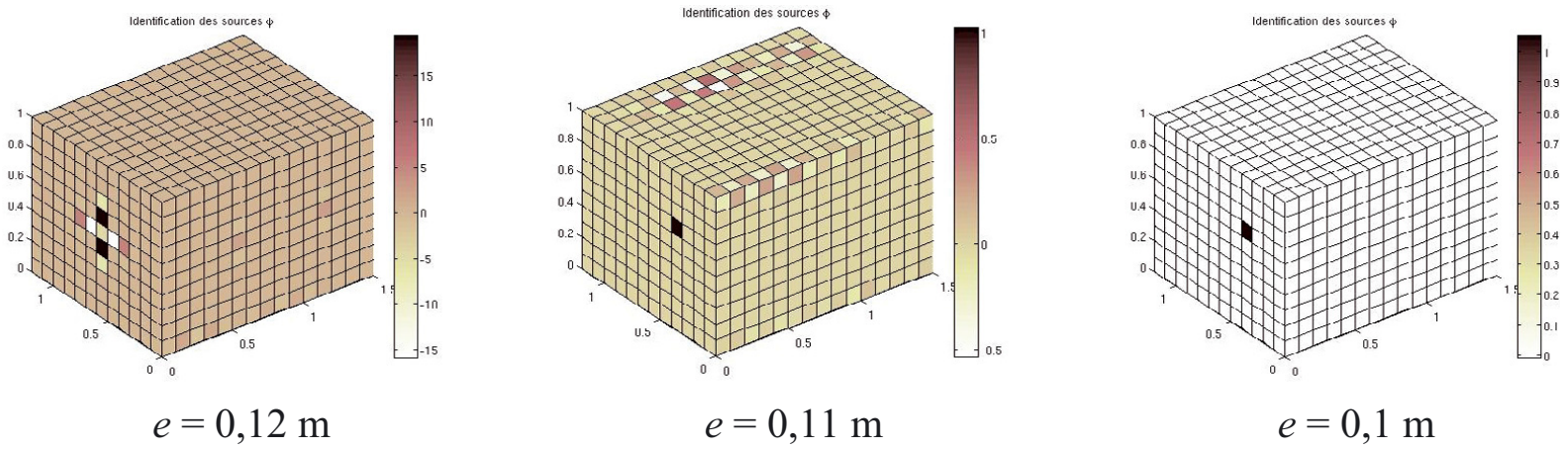

Fig. 6. Sensibilité à l'écartement entre les microphones.
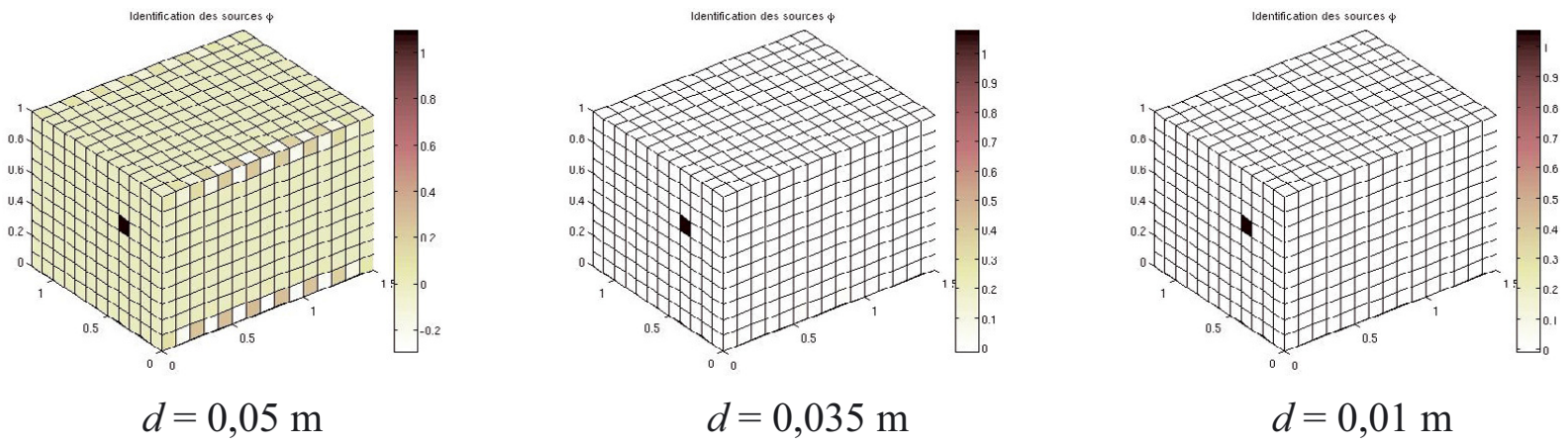

Fig. 7. Sensibilité à la distance entre les surfaces et les microphones.

et quantification de la source de $80 \mathrm{~mm}$ sans intrusion d'erreur à $35 \mathrm{~mm}$ à la suite de l'intrusion.

Malgré ces légères modifications des résultats après l'intrusion d'erreur de mesure aux données, la MES inverse localise bien la source acoustique, les résultats trouvés sont parfaits et robustes d'où sa validation.

\subsection{Validation MES/FEM}

Les méthodes de type éléments finis sont certes limitées en fréquence puisqu'elles nécessitent une discrétisation en rapport avec la longueur d'onde considérée. Cependant, il est possible de réaliser des moyennes sur ces solutions exactes afin de décrire les comportements asymptotiques qui leur sont associés.
Dans ce contexte, on a élaboré le même modèle sur un logiciel d'éléments-finis (COMSOL) en injectant la même puissance sur la source, puis on utilise les données récupérées (densité d'énergie $W$ et intensité $I$ ) et moyennées pour alimenter la MES inverse.

On cherche alors dans cette section à valider la MES pour un modèle comportant une source surfacique, plusieurs sources surfaciques ou une plaque vibrante de type A-A (appuyée-appuyée).

\subsubsection{Modèle à une source surfacique}

Le modèle est identique à celui développé en MES directe comportant une source en $(0,0,39,0,66)$ avec une 

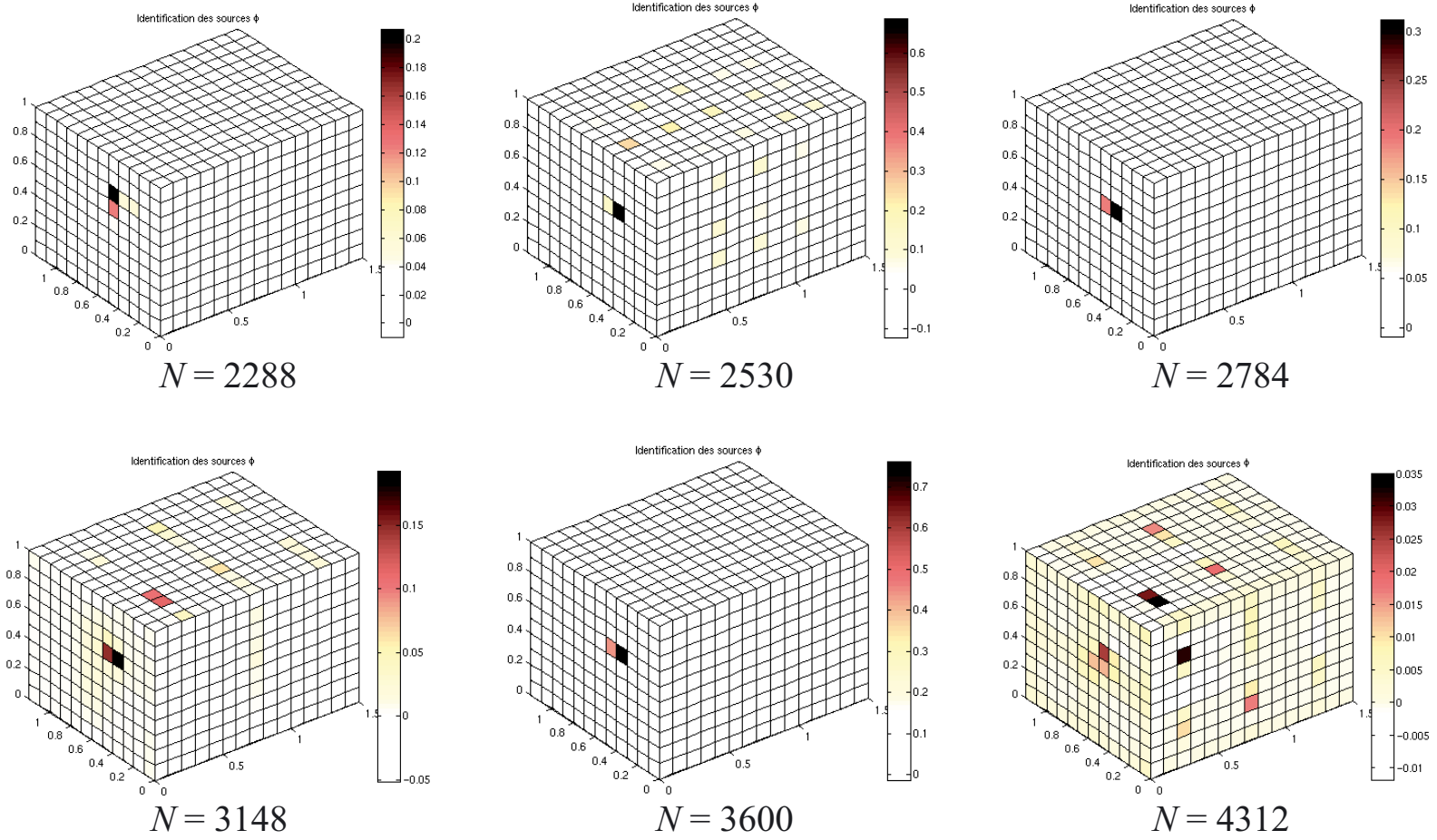

Fig. 8. Sensibilité au nombre de points de calcul $N$ pour le modèle à une source.

puissance injectée de $1 \mathrm{~W} \cdot \mathrm{m}^{-2}$ et une absorption au niveau des surfaces de 0,3 . La bande de fréquence étudiée est une octave de fréquence centrale $f_{\mathrm{c}}=1000 \mathrm{~Hz}$. La figure 8 affiche les résultats trouvés pour différents nombres de points de calcul.

On remarque qu'à partir de 2288 points de calcul, on pourra localiser la source selon des valeurs précises de $N$ $(2288,2530,2784$ ou 3600$)$ mais il reste de bien trouver la puissance injectée égale à $1 \mathrm{~W} \cdot \mathrm{m}^{-2}$. Il y a donc deux nombres de $N$ pour lesquels on s'approche de cette valeur pour $N=2530$ et $N=3600$ dont ce dernier est le plus clair du point de vue localisation. Pour justifier notre choix, on a utilisé un terme de précision qui est présenté comme suit :

$$
P=\frac{\sum_{i=1}^{N_{\mathrm{e}}}\left(\phi_{i}-\phi_{i}^{\text {exact }}\right)^{2}}{N_{\mathrm{e}}}
$$

où $\phi_{i}$ est le vecteur flux obtenu par FEM pour l'ième maille et $\phi_{i}^{\text {exact }}$ est le vecteur flux exact. On calcule alors la précision pour chacun des résultats pour voir lequel possède la plus faible valeur de $P$, et on répète tout ça en modifiant la bande de fréquences pour $f_{\mathrm{c}}=1500 \mathrm{~Hz}$ et $f_{\mathrm{c}}=2000 \mathrm{~Hz}$.

Tous ces résultats sont résumés dans la figure 9 qui illustre la sensibilité de la MES inverse à la fréquence centrale $f_{\mathrm{c}}$ et au nombre des points de calcul $N$.

On remarque que 3600 représente la valeur la plus stable de $N$ puisque la valeur de la précision trouvée est identique et est la plus petite pour différentes valeurs de la fréquence centrale, d'où la validation de notre choix pour $N=3600$ points.

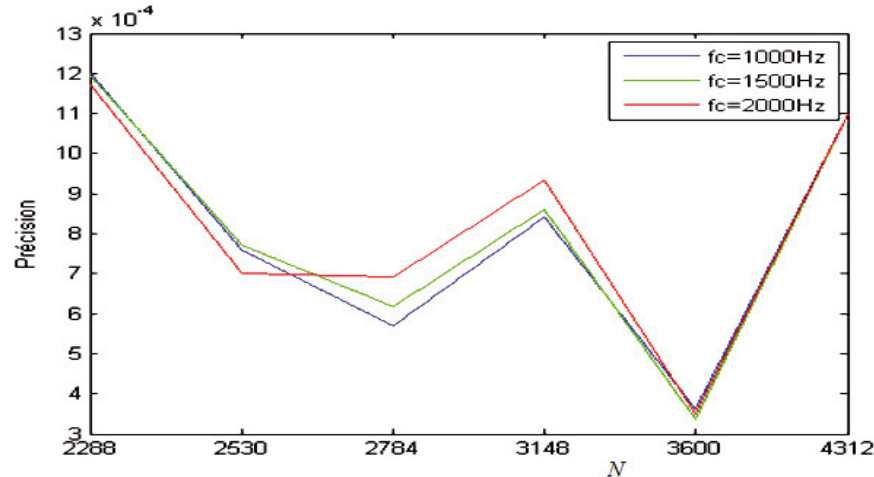

Fig. 9. Sensibilité à la valeur de la fréquence centrale $f_{c}$ et au nombre de points de calcul $N$.

D'autre part, il apparaît que la courbe d'évolution de $P$ en fonction de $N$ pour $f_{c}$ égale à $1000 \mathrm{~Hz}$ est la plus basse de point de vue précision, ce qui nous ramène à travailler dans ce qui suit avec une fréquence centrale de $1000 \mathrm{~Hz}$.

\subsubsection{Modèle à deux sources surfaciques}

Le modèle à deux sources comporte comme l'indique son nom deux sources acoustiques placées sur la surface de la cavité aux coordonnées $(0,0,39,0,66)$ et $(0,8,0,0,2)$. La même puissance injectée de $1 \mathrm{~W} . \mathrm{m}^{-2}$ est appliquée aux deux sources. L'absorption est maintenue toujours égale à 0,3 et la fréquence centrale est donc égale à $1000 \mathrm{~Hz}$. 

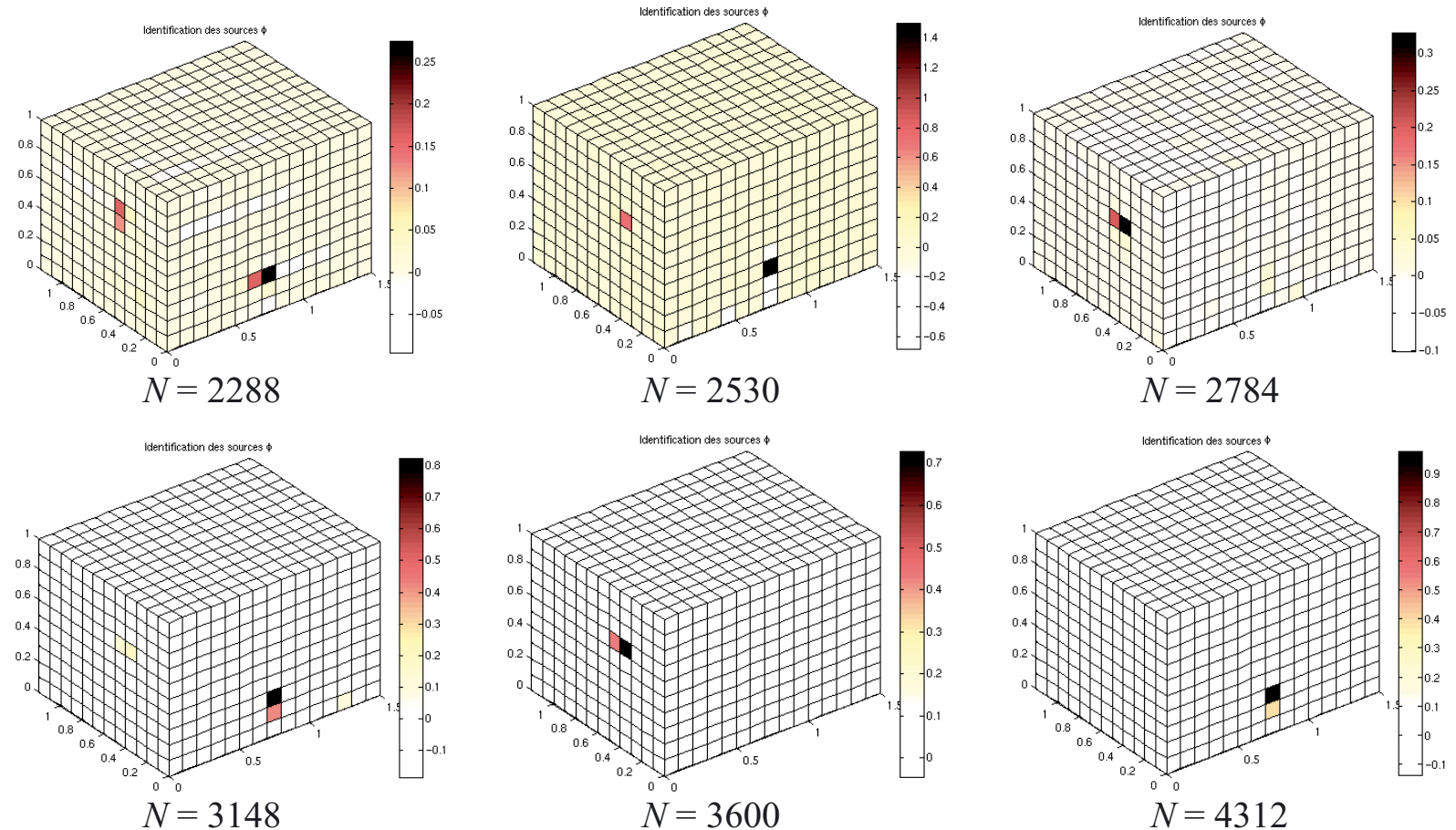

Fig. 10. Sensibilité au nombre de points de calcul $N$ pour le modèle à deux sources.

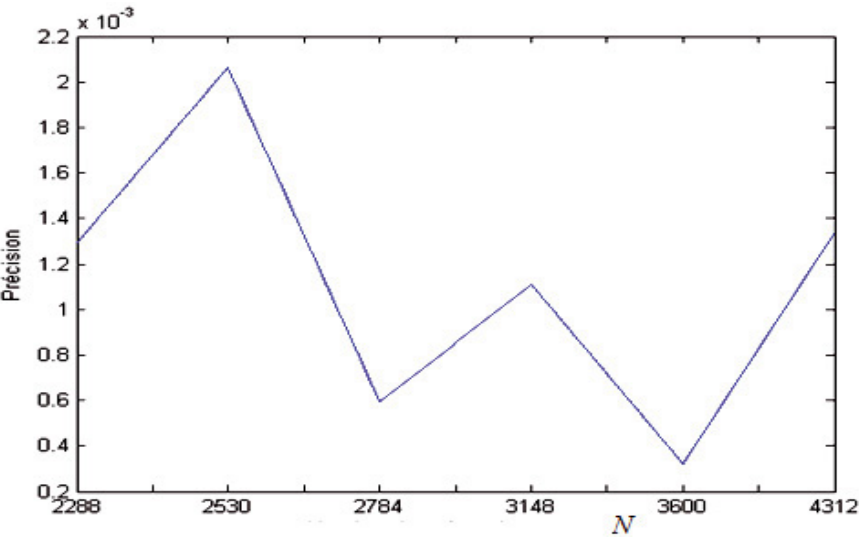

Fig. 11. Évolution de la précision en fonction de $N$.

Les résultats trouvés alors pour ce modèle sont présentés dans la figure 10 .

Pour un nombre de points de calcul égal à 2288 ou 2530, les deux sources sont localisées mais elles possèdent deux valeurs de puissance injectée différentes. La plus intéressante de ces deux résultats est pour $N=2530$ où les valeurs de la puissance injectée se rapprochent de $1 \mathrm{~W} \cdot \mathrm{m}^{-2}\left(0,7 \mathrm{~W} \cdot \mathrm{m}^{-2}\right.$ pour la première source et $1,4 \mathrm{~W} \cdot \mathrm{m}^{-2}$ pour la deuxième). Pour les autres valeurs de $N(2784,3148,3600$ et 4312$)$, la première ou la deuxième source seulement sera localisée avec des valeurs de la puissance proches de $1 \mathrm{~W} \cdot \mathrm{m}^{-2}$. D'après la figure 11 qui décrit l'évolution de la précision en fonction du nombre $N$, on remarque qu'on obtient toujours une faible valeur de précision pour $N$ égal à 3600 points.

\subsubsection{Modèle à une plaque vibrante de type $A-A$}

Ce modèle comporte une plaque de type AppuyéeAppuyée (A-A) placée sur la surface de la cavité et couplée avec le fluide. Cette plaque est de dimensions $0,2 \times 0,2$ et centrée sur le point $(0,0,25,0,75)$ où on applique une force qui fait vibrer la plaque selon des modes propres bien précis comme présentés dans [12]. Les résultats trouvés sont résumés dans la figure 12 .

On trouve deux bonnes identifications de l'emplacement de la plaque pour $N$ égal à 2288 et 3600 points sans quantifier la puissance injectée où les valeurs trouvées des flux restent faibles par rapport à $1 \mathrm{~W} \cdot \mathrm{m}^{-2}$. Pour les autres nombres de points de calcul, la plaque reste non identifiable.

\section{Conclusion}

Le but de cet article est de montrer la possibilité d'utiliser la méthode énergétique simplifiée MES pour localiser des sources acoustiques surfaciques à partir de mesures effectuées dans une cavité. Cette méthode convient bien avec la plage des moyennes et hautes fréquences. La MES inverse est validée pour la détection d'une source. En fait, il apparaît qu'elle est faisable et robuste après l'intrusion d'erreurs au niveau des données. Pour les modèles à plusieurs sources, la MES inverse est validée du point de vue localisation mais elle reste un peu incertaine en ce qui concerne la quantification des valeurs exactes des puissances injectées. 

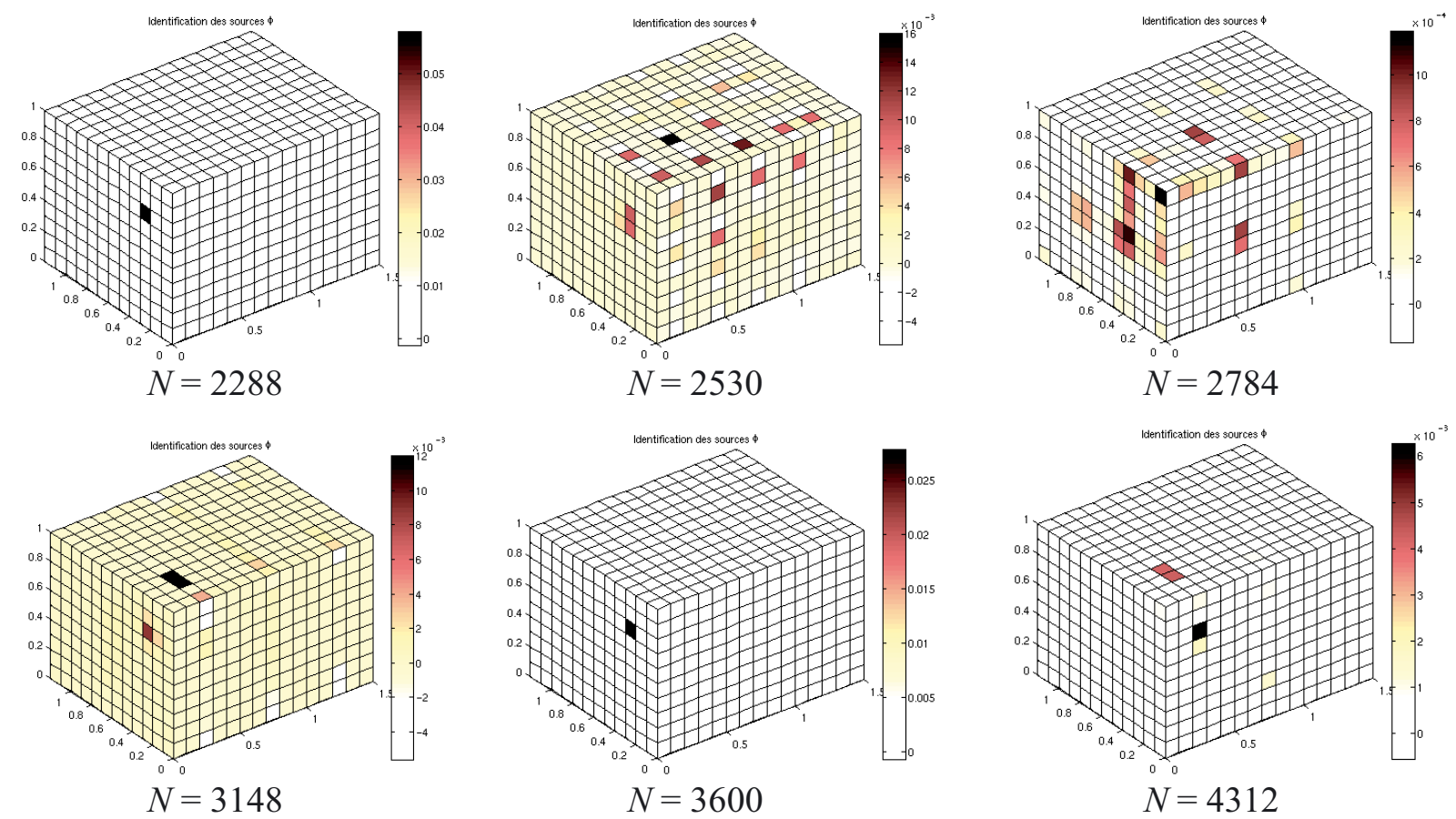

Fig. 12. Sensibilité au nombre de points de calcul $N$ pour le modèle de plaque.

En effet, si le système est excité par une distribution dense de sources, la divergence des fonctions de Green énergétiques au point d'excitation est problématique. De plus, la phase entre les sources peut jouer un rôle important. Enfin, dans certains cas où le champ direct constitue une part non négligeable du champ total, on souhaiterait pouvoir modéliser le champ direct de façon plus précise qu'avec les seules fonctions de Green énergétiques.

Pour y remédier, on essayera d'utiliser une méthode hybride [13] où le champ direct sera modélisé avec une méthode cinématique alors que le champ réverbéré est modélisé par une méthode énergétique.

\section{Références}

[1] T. Schmitt, Modélisation des transferts acoustiques en moyennes et hautes fréquences par méthode énergétique, Thèse de doctorat, École Centrale de Lyon, 2004

[2] P. Hardy, Méthodes énergétiques simplifiées en moyennes et hautes fréquences : Théorie et applications industrielles, Thèse de doctorat, École Centrale de Lyon, 2005

[3] R.S. Langley, On the vibrational conductivity approach to high frequency dynamics for the dimensional structural components, J. Sound Vib. 182 (1995) 637-657

[4] P. Moron, Mise en œuvre de méthodes énergétiques dans l'étude de comportement vibratoire de structures complexes dans le domaine des moyennes et hautes fréquences, Thèse de doctorat, École Centrale de Lyon, 1998

[5] A. Le Bot, A vibroacoustic model for high frequency analysis, J. Sound Vib. 211 (1998) 537-554

[6] R.N. Miles, Sound field in a rectangular enclosure with diffusely reflecting boundaries, J. Sound Vib. 92 (1984) 203-226

[7] M.N. Ichchou, L. Jezequel, New advances in energy models for the non modal vibration and noise prediction, Transworld Research Network

[8] Y. Lase, M.N. Ichchou, L. Jezequel, Energy analysis of bars and beams: theoretical formulations, J. Sound Vib. 192 (1996) 281-305

[9] M.N. Ichchou, L. Jezequel, Comments on simple models of the energy flow in vibrating membranes and transversely vibrating plates, J. Sound Vib. 195 (1996) 679-685

[10] M.N. Ichchou, A. Le Bot, L. Jezequel, Energy models of one-dimensional multi-propagative systems, J. Sound Vib. 201 (1997) 535-554

[11] H. Kuttruff, A simple iteration scheme for the computation of decay constants in enclosures with diffusely reflecting boundaries, J. Acoustical Soc. Am. 98 (1995) 288-293

[12] Springer Handbook of Acoustics, New York, 2007

[13] M.J. Smith, A hybrid energy method for predicting vibrational response of point loaded plates, J. Sound Vib. 202 (1997) 375-392 